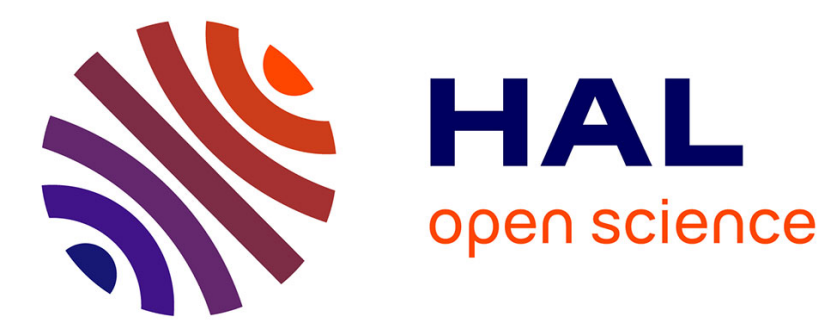

\title{
Considérations sur les anthroponymes phéniciens transcrits en grec dans les inscriptions bilingues gréco-phéniciennes
}

Maria Bianco

\section{- To cite this version:}

Maria Bianco. Considérations sur les anthroponymes phéniciens transcrits en grec dans les inscriptions bilingues gréco-phéniciennes. Semitica et Classica, 2015, 8, pp.53-62. 10.1484/J.SEM.5.109177 . hal01631207

\section{HAL Id: hal-01631207 \\ https://hal.science/hal-01631207}

Submitted on 11 Jan 2018

HAL is a multi-disciplinary open access archive for the deposit and dissemination of scientific research documents, whether they are published or not. The documents may come from teaching and research institutions in France or abroad, or from public or private research centers.
L'archive ouverte pluridisciplinaire HAL, est destinée au dépôt et à la diffusion de documents scientifiques de niveau recherche, publiés ou non, émanant des établissements d'enseignement et de recherche français ou étrangers, des laboratoires publics ou privés. 


\section{CONSIDÉRATIONS SUR LES ANTHROPONYMES PHÉNICIENS TRANSCRITS EN GREC DANS LES INSCRIPTIONS BILINGUES GRÉCO-PHÉNICIENNES*}

\section{Introduction}

L'élaboration d'une écriture alphabétique a dû connaître une longue période de gestation avant d'aboutir à la forme qu'Hérodote appelle phénicienne'. Cette attribution s'est cristallisée pour devenir une tradition, dont le récit de l'historien a fixé le souvenir, justement, par écrit. Ce furent les Cadméens eux-mêmes, une fois installés en Béotie à la suite de Cadmos, qui, au fur et à mesure qu'ils changèrent leur langue, modifièrent aussi la forme originaire des lettres rapportées de Phénicie. Les Ioniens prirent la suite, en adaptant les phoinikeia grammata à la langue grecque ${ }^{2}$. Ainsi l'anecdote s'ancre-t-elle au mythe.

Faute de documentation, l'on n'est pas en mesure de déterminer quand et surtout où les Phéniciens ont pu enseigner les phoinikeia grammata aux Grecs. Si l'on cherche un lieu où les deux peuples ont pu vivre côte à côte pendant un temps raisonnablement suffisant à la transmission d'un tel savoir, l'archéologie nous conduit à Chypre. Pourtant, bien que les Phéniciens y ont été établis au moins depuis le $\mathrm{IX}^{\mathrm{e}}$ siècle av. J.-C., notamment dans le centre de Kition ${ }^{3}$, une écriture alphabétique en grec n'est attestée sur l'île qu'à partir du VI siècle av. J.-C. Par contre, à notre connaissance, les Grecs commencèrent à utiliser les phoinikeia grammata dès le début du VIII ${ }^{\mathrm{e}}$ siècle, au moins. L'inscription sur un vase de la tombe 482 de la nécropole latiale de l'Osteria dell'Osa ${ }^{4}$ est datée du 770 av. J.-C., et elle représente, à ce jour, le plus ancien exemple connu d'écriture alphabétique grecque. Quelques décennies plus tard, l'inscription de l'oenochoè du Dipylon (740 av. J.-C.) et celle de la coupe de Nestor de Pithécusses (assignée au dernier quart du VIII ${ }^{e}$ s. av. J.-C.) témoignent d'une étape déjà avancée de la réception de l'alphabet, s'agissant dans l'un et dans l'autre cas, de mise en vers. Ainsi, les Grecs adaptèrent-ils très tôt les signes alphabétiques à leur langue. Plus tard, quand des Phéniciens s'installèrent de façon plus durable parmi les Hellènes, des éléments de la langue phénicienne ellemême furent exprimés au moyen des lettres que jadis ils leur avaient enseignées. En l'occurrence, on songe ici aux anthroponymes phéniciens adaptés en grec dans un petit groupe d'inscriptions bilingues

\footnotetext{
${ }^{*}$ Ce travail est inscrit dans l'axe thématique Identités du programme scientifique du Labex ARCHIMEDE, programme «Investissement de l'avenir » IA- ANR-11-LABX-0032-01. Je remercie M. G. Amadasi Guzzo, C. Bonnet, R. Catling, F. Briquel-Chatonnet, M. Egetmeyer, E. Perrin-Saminadayar qui ont relu tout ou partie de cet article pour les remarques qu'ils ont bien voulu m'adresser.

${ }^{1}$ Hérodote V, 57-58.

${ }^{2}$ Hérodote V, 58 : «puis, à mesure que le temps passait, en même temps qu'ils changeaient de langue, les Cadméens changèrent aussi la forme des caractères. La plupart des régions d'alentour étaient habitées à cette époque par des Grecs de race ionienne; ils empruntèrent les lettres aux Phéniciens qui les leur avaient enseignées, et les employèrent légèrement modifiées » (éd. et tr. par Ph.-E. Legrand, Paris 1946).

${ }^{3}$ DCPhP : 248-249

${ }^{4}$ La Regina 1989-1990 : 83-88.
} 
gréco-phéniciennes, qui remontent à la période située entre le $\mathrm{IV}^{\mathrm{e}}$ et le $\mathrm{II}^{\mathrm{e}}$ siècle av. J.-C. Elles proviennent de la Grèce continentale et insulaire. Si les inscriptions bilingues gréco-phéniciennes ont déjà fait l'objet de plusieurs études, un recueil complet de ces documents n'a pas encore vu le jour. Elles font pour l'heure partie du corpus des inscriptions prises en compte dans le cadre d'un doctorat dont le but est de reconstruire les mailles du réseau des relations entre les Grecs et les Phéniciens par le biais des témoignages épigraphiques ${ }^{5}$. Qu'il s'agisse de dédicaces, ou d'épitaphes, les inscriptions bilingues fournissent de nombreuses attestations onomastiques, présentes dans les deux versions des textes, grecque et phénicienne.

Le passage d'une langue à l'autre peut être réalisé de différentes façons. Si l'on imagine un segment représentant tous les mécanismes d'adaptation possibles pour un anthroponyme migrant d'une langue à une autre, aux deux extrémités de celui-ci on placerait les cas de traduction et les cas de transcription phonétique pure. Au centre, en ce qui concerne le phénicien, pour lequel, comme pour toute langue sémitique, les noms théophores représentent la majorité des noms personnels, se placent les théonymes interprétés et ensuite adaptés ; les mécanismes d'adaptation sont sélectionnés à chaque fois par la langue d'arrivée. Une étude d'ensemble des différents types d'adaptation onomastique dans les inscriptions bilingues gréco-phénicienne a été présenté il y a plus de vingt ans ${ }^{6}$. Parmi les processus linguistiques envisagés, il a paru important de mettre ici en lumière les mécanismes qui président aux transcriptions des noms phéniciens en grec. De Chypre à Athènes, en faisant escale à Cos, des noms phéniciens sont passés à travers le filtre d'une transcription phonétique, censée reproduire le son originaire du mot de départ. Ce processus s'est achevé par l'insertion du nom phénicien dans les catégories morphologiques du grec, sauf dans l'un des cas ici examinés.

\section{Les noms bâtis sur le théonyme ssm : CIS I, 95 ; ICS 168 ; ICS 216}

En dépit d'une étymologie encore obscure, le théonyme $s s m^{7}$ s'avère être un élément assez productif dans le domaine de l'anthroponymie. Il apparaît déjà à date ancienne dans la documentation ougaritique $^{8}$, et il est attesté à Chypre avec le plus grand nombre d'occurrences en milieu phénicien ${ }^{9}$. Trois inscriptions bilingues trouvées sur l'île nous offrent le cas de noms théophores bâtis à partir du théonyme $s s m^{10}$ et transcrits en grec, en écriture à la fois syllabique et alphabétique.

\footnotetext{
${ }^{5}$ Maria Bianco, «Les Phéniciens et les Grecs en Méditerranée : étude de leurs relations à travers les témoignages épigraphiques ( $\mathrm{du} \mathrm{V}^{\mathrm{e}}$ au $\mathrm{II}^{\mathrm{e}}$ av. J.-C.) », thèse de doctorat sous la direction d'Éric PerrinSaminadayar et de Corinne Bonnet, à l'université Paul-Valéry, Montpellier.

${ }^{6}$ Amadasi Guzzo \& Bonnet $1991: 1-21$

${ }^{7}$ Sur cette divinité, voir Caquot \& Masson 1968 : 317-320 ; DCPhP :396 ; Lipiński 1995 : 292-296.

${ }^{8} \mathrm{KTU} 4.170,18$ et $K T U 1.75,12$.

${ }^{9}$ Pour les attestations, voir Benz 1972 : 368.

${ }^{10}$ Cf. CIS I, 46 ; CIS I, 49 ; CIS I, 53 ; CIS I, 93 ; CIS I, 95 ; ICS 168 et ICS 216.
} 
Examinons d'abord une inscription de Lapéthos ${ }^{11}$. Il s'agit d'une dédicace gravée sur un rocher ; elle présente une originalité remarquable, puisqu'elle est dédiée conjointement à un dieu et un roi. Tous les éditeurs s'accordent à dater l'inscription de la fin du $\mathrm{IV}^{\mathrm{e}} \mathrm{s}$. av. J-C. ou du début du III ${ }^{\mathrm{e}} \mathrm{s}$. av. J.-C. Le roi en question serait alors Ptolémée ${ }^{\text {er }}$ Sôter $^{12}$ :

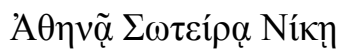

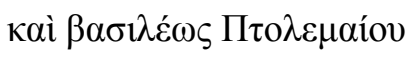

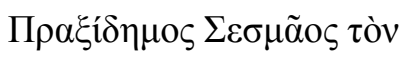 \\ $\beta \omega[\mu o ̀] v \dot{\alpha} v \varepsilon ́ \theta[\eta \kappa] \varepsilon v$ \\ $\dot{\alpha} \gamma \alpha[\theta] \tilde{\eta} \iota \tau \hat{\chi} \chi 1$.
}

l'nt [m]'z hyym

wl'dmlkm ptlmyš

b'lšlm bn [s]smy

yqdš [']t mzbḥ

$[1] \mathrm{mzl} \mathrm{n}$ 'm
« À Athèna Sôteira Nikè et au roi Ptolémée Praxidèmos fils de Sesmâs a dedié cet autel. À la bonne fortune »

«À ' $n t$ rempart des vivants et à Ptolémée seigneur des rois $b$ 'šlm fils de ssmy a consacré cet autel. À la bonne fortune ${ }^{13}{ }$,

Le patronyme du dédicant est enregistré dans la partie grecque de l'inscription comme $\Sigma \varepsilon \sigma \mu \tilde{\alpha}$ os. Il s'agit du génitif d'un nom qu'on reconstruit au nominatif comme ${ }^{*} \Sigma \varepsilon \sigma \mu \alpha \tilde{\alpha} \varsigma$. L'anthroponyme qui lui correspond en phénicien est en partie restitué, mais avec un fort degré de probabilité, comme $[s]$ smy; il s'agit de la forme hypocoristique ${ }^{14}$ d'un nom théophore construit sur le théonyme ssm, en l'occurrence un hypocoristique pourvu du suffixe en yod. La transcription en grec reproduit le thème nominal du nom divin selon une séquence Consonne-Voyelle-Consonne-Consonne (CVCC). L'on verra que la restitution du même théonyme au moyen du syllabaire chypriote ne contredit pas ce schéma nominal.

\footnotetext{
${ }^{11}$ CIS I, $95=$ KAI $42=$ RÉS 1515

${ }^{12}$ Près de Tyr, dans ce qu'on appelle « la grotte de Wasța », c'est encore Ptolémée I ${ }^{\text {er }}$ Sôter qui fait l'objet d'une dédicace en grec, en association avec la déesse Aphrodite ( $c f$. CIS I, 6). L'étude de C. Bonnet sur l'inscription de la grotte de Wasța en offre une nouvelle et convaincante interprétation, qui tire au clair l'association inhabituelle d'un roi et d'une divinité comme destinataires d'une dédicace (Bonnet $2004: 125-140$ ).

${ }^{13}$ Pour la traduction ici reproduite, voir Bonnet $2004: 136$.

${ }^{14}$ Un aperçu sur les noms hypocoristiques dans Benz $1972: 232-235$.
} 
La légende monétaire d'un lot de monnaies provenant de Marion nous fait connaître l'émission d'un roi phénicien qui aurait gouverné la ville pendant la première moitié du $\mathrm{V}^{\mathrm{e}} \mathrm{s}$. av. J.-C. ${ }^{15}$ :
(d.) (= gén.) sa-sa-ma-o-se-to-ka-sa-to-ro
«De Sasmâs, (fils) de Doxandros/De
(r.) (= nom.) ma-rie-u-se
Marion»

(d.) $\Sigma \alpha \sigma \mu \tilde{\alpha} 0 \varsigma \Delta \mathrm{o} \xi \alpha \dot{\alpha}(v) \delta \rho \omega$

(r) Mapızús

Le nom du souverain est attesté au génitif avec l'orthographe sa-sa-ma-o-se, qui est à transcrire $\Sigma \alpha \sigma \mu \tilde{\alpha}$ os, comme le propose déjà O. Masson ${ }^{16}$. Cette transcription confirme la structure CVCC pour la prononciation du théonyme $s s m$, attestée par la forme en grec alphabétique de Lapéthos.

Les transcriptions en grec du théonyme ssm autorisent quelques remarques intéressantes : à Lapethos, $\Sigma \varepsilon \sigma \mu \tilde{\alpha}$ os est le génitif d'un nominatif que l'on reconstruit comme $* \Sigma \varepsilon \sigma \mu \tilde{\alpha} \varsigma$; de même, sur les pièces de Marion, pour le génitif $\Sigma \alpha \sigma \mu \tilde{\alpha} o \varsigma$, on reconstruit aisément le nominatif * $\Sigma \alpha \sigma \mu \alpha \tilde{\alpha} \varsigma$. En fait, tant à Lapéthos qu'à Marion, ce nom théophore a été inséré dans la déclinaison des noms en -ã, , $\tilde{\alpha}(F) o \varsigma(>\tilde{\alpha} 0 \varsigma)$, qu'on pourrait qualifier de locale, c'est-à-dire de chypriote, du fait que, comme $\mathrm{O}$. Masson ${ }^{17} 1$ 'a bien démontré, il s'agit d'une flexion nominale très fréquente à Chypre pour les noms propres, qu'ils soient grecs ou étrangers.

À ces deux exemples on peut ajouter le patronyme $\Sigma \varepsilon \sigma \mu \alpha$ íov qui apparaît dans une inscription provenant de la colonie sidonienne de Marisa en Palestine ${ }^{18}$, datant de la seconde moitié du $\mathrm{I}^{\mathrm{er}} \mathrm{s}$. av. J.C. ${ }^{19}$. Les équivalences ici ne posent pas de problèmes, le nominatif attendu étant ${ }^{*} \Sigma \varepsilon \sigma \mu \alpha o \zeta$, tandis que l'équivalent phénicien attendu est ssmy. Il existe encore deux témoignages très importants pour la présente enquête. Ils se situent dans un contexte totalement sémitique : dans le premier livre des Chroniques, parmi les descendants de la tribu de Juda, apparaît le nom Sismay ${ }^{20}$, qui représente encore une fois la forme hypocoristique ssmy du phénicien. D'autre part, un document assyrien datant du règne d'Assurbanipal (668-626 av. J.-C.) nous fait connaître un certain Ša-aš-ma-a, qu'A. Caquot et O. Masson qualifient de probablement araméen ${ }^{21}$ et qui a dû porter un nom proche de *ssm'.

\footnotetext{
${ }^{15}$ Gjerstad 1946 : 21-24 ; ICS 168. Il est à noter que deux exemplaires de cette série présentent sur le verso deux lettres phéniciennes, à savoir $m l$, ce qui étaye l'hypothèse que le roi battant la monnaie était un Phénicien, $c f$. Masson \& Sznycer 1972 : 79-81; Egetmeyer $2010: 715-716$.

${ }^{16}$ Masson $1961: 182$.

${ }^{17}$ Masson $1958: 67-72$

${ }^{18}$ OGIS 593. L'endroit était une « colonie sidonienne », cf. Caquot \& Masson $1968: 318$.

19 Cf. Lagrange 1902 : 500.

${ }^{20} 1$ Chr. $2: 40$

${ }^{21}$ Caquot \& Masson 1968 : 318.
} 
Les remarques qui précédent permettent de revenir sur l'inscription ICS 216, qui atteste le nom théophore 'bdssm, rendu en grec syllabique par a-pa-sa-so-mo-se, transcrit $\mathrm{A} \psi \alpha \sigma \omega \mu \mathrm{\alpha} \varsigma$ dans l'édition d'O. Masson ${ }^{22}$. En 1885, dans un sanctuaire d'Apollon près de Tamassos (Phrangissa), a été trouvée une base de statue en marbre inscrite ${ }^{23}$, offerte par un Phénicien à Apollon Alasiotas, ršs 'lhyts dans la version phénicienne :

$$
\begin{aligned}
& \text { bymm 10+6 lyrh p'lt bš[n-] } \\
& \text { t 10+7(?) lmlk mlky[tn mlk k-] } \\
& \text { ty w'dyl sml 'z 'š ytn 'b- } \\
& \text { dssm bn...l'dny lršp '- } \\
& \text { lhyts hndr 'š ndr kšm' } \\
& \text { h' ql ybrk }
\end{aligned}
$$$$
\text { a-ti-ri-a-se } \| \text { o-nu-to-ne-to }
$$$$
\text { ke-ne | a-pa-sa-so-mo-se | o-sa }
$$$$
\text { ma-wo-se | to-i-a-[po-lo]-ni-to-i }
$$$$
\text { a-la-si-o-ta-i | i-tu-ka-i }
$$

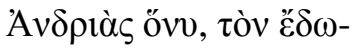$$
\kappa \varepsilon v \text { A } \psi \alpha \sigma \mu \circ \varsigma \text { ó } \Sigma \alpha-
$$

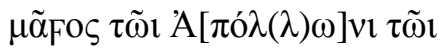$$
\text { A } \lambda \alpha \sigma i \omega ́ \tau \alpha \imath i(v) \tau u ́ \chi \alpha \iota
$$

«Le $16^{\mathrm{e}}$ jour du mois de $p$ 'lt, en l'année 17 du roi $m l k y[t n$, roi de Ki]tion et Idalion, cette statue est ce qu'a donné 'bdssm, fils de ..., à son seigneur, à řs̆p 'lhyts, le vœu que lui (le dieu) a voué parce qu'il a écouté sa voix. Puisse-t-il le bénir !»

«Cette statuette, qu'a offerte Apsasmos, le (fils) de Samâs, à Apollon Alasiôtas. À la (Bonne) Fortune »

Le nom du dédicant, 'bdssm dans la partie phénicienne de l'inscription, est rendu en écriture syllabique comme a-pa-sa-so-mo-se. Or, sur la base des considérations faites ci-dessus, nous sommes

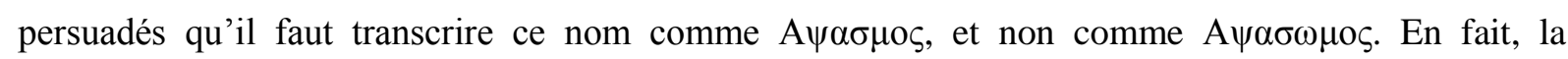
transcription $\mathrm{A} \psi \alpha \sigma \mu \mathrm{o}$ fait correspondre, une fois de plus, le théonyme ssm à la structure CVCC. Outre cette reconstitution du nom, on peut observer quelques spécificités phonétiques très intéressantes : la dentale de 'bd s'assimile à la sibilante initiale du théonyme ssm et, en conséquence, la rencontre entre la labiale et la sibilante peut être rendue en grec par la consonne double $\psi$; l'anthroponyme est ensuite inséré dans la déclinaison thématique, marquée par la terminaison -oc du nominatif. Dans son recueil d'inscriptions chypriotes syllabiques, O. Masson ${ }^{24}$ propose une reconstruction différente du nom du dédicant, qu'il transcrit comme A $\psi \alpha \sigma \omega \mu \rho \varsigma$; il suit en cela l'interprétation du nom avancée par Ph. Berger en $1887^{25}$. Il est vrai que la structure du syllabaire

\footnotetext{
${ }^{22}$ Masson $1961: 227$; $c f$. aussi Egetmeyer 2010 : 813-814.

${ }^{23}$ ICS 216.

${ }^{24}$ Masson $1961: 227$.

${ }^{25}$ Berger 1887 : 195 n. 1.
} 
s'accorde avec les deux reconstitutions proposées, mais en faveur de la structure CVCC, qu'on a privilégiée ici, il existe les témoignages parallèles de CIS I, 95, OGIS 593 et 1 Chr. 2 :40, tandis que, pour une structure de type CVCVC, on n'a pas d'autre attestation. De plus, dans son étude, $\mathrm{Ph}$. Berger commet une erreur : il compare 'bdssm à d'autres anthroponymes phéniciens, comme b'lytn, où l'on a affaire à un nom divin suivi d'une forme verbale au Qal parfait $3^{\mathrm{e}}$ pers. s. m. : /Apsasōm-/ comme /Ba'al-yatōn/ «Ba'al a donné ». À la base de la transcription phonétique que Berger suggère, il y a donc une erreur d'interprétation du mot ssm, que le savant a interprété comme une forme verbale, alors qu'il s'agit d'un théonyme.

Les formes que l'on a analysées précédemment représentent le nom du dieu ssm dans ses versions hypocoristiques; la seule occurrence faisant exception est la dernière, attestée par l'inscription de Phrangissa, où $\mathrm{A} \psi \alpha \sigma \mu \mathrm{s}$ renferme la forme simple du théonyme, qui constitue le deuxième élément d'un nom théophore régi par le mot 'bd: 'bdssm «serviteur du dieu $s s m$ », à vocaliser Sasm.

La même dédicace de Tamassos atteste un autre anthroponyme qui mérite quelques considérations. La version grecque de l'inscription fournit le patronyme du dédicant au génitif, à savoir sa-ma-wo-se, que l'on n'hésitera pas à transcrire $\Sigma \alpha \mu \alpha \tilde{\alpha} F o \zeta$. On est face à un autre cas de nom étranger qui est absorbé par la déclinaison chypriote des noms en -ã ; c'est pourquoi on envisagera aisément un nominatif $* \Sigma \alpha \mu \tilde{\alpha}$. Le nom phénicien qui lui correspondrait ne nous est pas préservé dans la partie phénicienne de l'inscription qui, en ce point, est lacunaire. Toutefois, il est fort probable que le père du dédicant s'appelât en phénicien $*^{s} m$ ', šmy, ou $\check{s} m$ ", une des formes hypocoristiques possibles construites sur $\breve{\mathrm{S} M} \ll$ nom $»^{26}$ ou $\breve{\mathrm{S}} \mathrm{M}^{e}$ « écouter $»^{27}$.

\section{Le cas de Mnḥm de ICS 215}

Une deuxième base en marbre, inscrite et dédiée à Apollon Heleitas/rš̌p 'lyyt, a été trouvée dans les mêmes circonstances que la précédente ${ }^{28}$, dans le temple d'Apollon à Phrangissa. L'inscription nous permet de dater l'offrande du règne du même roi $m l k y t n$, mais environ une décennie plus tard ${ }^{29}$.

\footnotetext{
${ }^{26} C f$. Benz $1972: 419$ et $D N W S: 1155-1159$.

${ }^{27} C f$. Benz 1972 : 419 et DNWS : 1164-1166.

${ }^{28}$ ICS 216.

${ }^{29}$ Pour la datation des deux monuments voir Masson $1961: 225$, n. 2
} 
sml 'z 'š ytn wyțn

' mnḥm bn bnḥdš bn mn-

ḥm bn 'rq l'dny 1[rš ]p

'lyyt byrh 'tnm bšnt

šlšm 20+10 lmlk mlkytn mlk

kty w'dyl kšm‘ ql ybrk

to-na-ti-ri-a-ta-ne I| to-nu | e-to-ke-ne

ka-se | o-ne-te-ke-ne \|| ma-na-se-se

o-no-me-ni-o-ne \| to-i-ti-o-i

to-i-a-pe-i-lo-ni || to-i-e-le-wi

ta-i $\|$ i-tu-ka-i

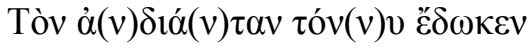

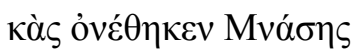

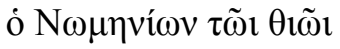

$\tau \tilde{\omega} \iota$ 'A $\pi \varepsilon i ́ \lambda \omega v \imath \tau \tilde{\omega} \iota{ }^{\circledR} \mathrm{E} \lambda \varepsilon F^{i}-$

$\tau \alpha l i(v) \tau u ́ \chi \alpha l$
«Cette statue est ce qu'a donné et érigé $m n h ̣ m$, fils de $b n h \underline{s}$, fils de $m n h \underline{m}$ fils de ' $r q$, à son seigneur, à $[r s ̌ s] p ~ ' l y y t$, au mois de'tnm, en l'année $30 \mathrm{du}$ roi Mlky[tn, roi de Ki]tion et Idalion, parce qu'il a écouté sa voix. Puisse-t-il le bénir !»

«Cette statuette (est ce) qu'a donné et dédié Mnáses, le (fils) de Noumenios, au dieu Apollon Heleitas. À la (Bonne) Fortune »

La dédicace a été faite par un Phénicien qui porte un nom, $m n h ̣ m$, attesté plusieurs fois en phénicien ${ }^{30}$ et dans d'autres langues sémitiques ${ }^{31}$. La forme est à interpréter comme un participe Piel dont la signification est « celui qui console ». La partie grecque syllabique de cette inscription livre, en correspondance de $m n h m$, la séquence ma-na-se-se. Des transcriptions alphabétiques différentes ont été proposées. Les premiers éditeurs ont opté pour la forme $\operatorname{M} \alpha \alpha \sigma(\sigma) \eta \varsigma$, qui est en fait la traduction grecque de la Septante de l'anthroponyme hébraïque $m n s ̌ h^{32}$. Cet anthroponyme n'est attesté qu'une seule fois en phénicien, à Éléphantine, sous la forme $m n \check{s} y^{33}$. Mnhlm et $m n \check{s} y$ étant des noms personnels différents, on ne leur attribuera pas la même forme d'adaptation en grec. En fait, tous deux sont des formes de participe Piel, mais le premier est bâti sur la racine $\mathrm{NHM}^{34}$, tandis que le deuxième est

\footnotetext{
${ }^{30}$ Benz $1972: 141$.

${ }^{31}$ Benz 1972 : 359-360

${ }^{32}$ Voir $I P N$ : 211 n. 1, 222.

${ }^{33}$ Benz 1972 : 142, 363-364.

${ }^{34}$ BDB s.v., $K B: 688$.
} 
construit sur la racine NŠY (hébreu NŠH${ }^{35}$, avec la signification de «celui qui fait oublier ». Néanmoins, bien qu'issus de racines différentes, ces deux anthroponymes pourraient être rapprochés au niveau sémantique ${ }^{36}$.

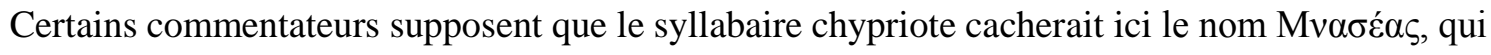
est assez bien attesté pour des Phéniciens à $\mathrm{Chypr}^{37}$, mais que dans la forme alphabétique ${ }^{38}$. Si tel était le cas, le dédicant aurait adopté un deuxième nom grec. Toutefois, à moins de supposer une erreur du lapicide, la séquence syllabique ma-na-se-se ne peut pas correspondre à la réalisation phonétique de

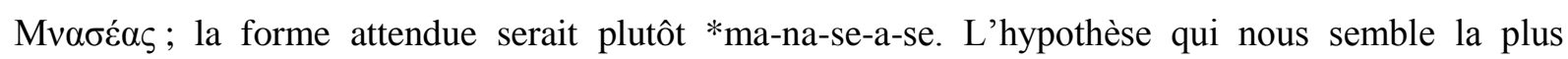
pertinente est celle que suggère O. Masson, qui édite le texte avec la transcription Mvó $\varsigma^{39}$. Ainsi, on aurait affaire ici presque à une transcription phonétique du nom phénicien suivant un critère d'assonance. L'on voit qu'en correspondance de la pharyngale ḥ, le grec présente le syllabogramme se-. Ce phénomène trahit la possibilité d'une aspiration de la sifflante en position intervocalique dans certaines circonstances dans le dialecte chypriote ${ }^{40}$. En examinant le corpus des noms phéniciens transcrits en grec, M. G. Amadasi ${ }^{41}$ fait remarquer que les pharyngales et les laryngales du phénicien ne sont jamais transcrites en grec, dans aucune position. Toutefois, à Chypre, à la suite de l'amuïssement de la sibilante, on a pu rendre la pharyngale phénicienne suivant une orthographe qui paraissait «correcte» (cf. dans ICS 216 'A $\lambda \alpha \sigma t \omega ́ \tau \alpha 1$, correspondant à 'lhyts du phénicien, qui évidemment est une transcription de l'adjectif grec tel qu'il était prononcé à Chypre). L'adaptation de cet anthroponyme phénicien s'est parachevée avec la création d'un nominatif en $-\eta \varsigma$, qui, s'il ne respecte pas la phonétique du terme sémitique qui est à sa base, obéit toutefois aux exigences syntaxiques de la phrase : le dédicant étant le sujet de l'action, la syntaxe demande un nominatif.

\section{La seule attestation de Mlkytn en grec : ICS 220}

En 1869, dans le temple d'Apollon à Idalion, a été mise au jour une base en marbre inscrite, dédiée à Apollon Amyklos/ršp mkl. Comme les deux bases provenant de Phrangissa, celle-ci devait soutenir une statue masculine; les statues ne nous sont pas parvenues, mais les deux textes de Phrangissa les mentionnent explicitement : $s m l$ en phénicien, àvoplás en grec. Par contre, ici, le mot $s m l$ est reconstitué sur la base du texte grec.

\footnotetext{
${ }^{35}$ BDB s.v., $K B: 728$.

${ }^{36}$ Les implications sémantiques envisageables pour $m n h ̣ m$ et $m n s ̌ h / m n s ̌ y$ ont été mises en lumière par F. Briquel-Chatonnet ( $c f$. Briquel-Chatonnet 1995 : 205).

${ }^{37}$ Masson 1968 :399, n. 1.

${ }^{38} \mathrm{Je}$ remercie M. Egetmeyer pour ces précisions.

${ }^{39}$ ICS 215. Cf. aussi Egetmeyer 2010 : 812-813.

${ }^{40}$ Morpurgo Davies 1988 : 113-124 ; Egetmeyer 2010 : 182

${ }^{41}$ Amadasi \& Bonnet 1991 : 3-4.
} 
[bymm? lyrḥ?] bšnt 'rb' 4 lmlk mlkytn m[lk] [kty w'dyl sml] 'z 'š ytn wyṭn' 'dnn b'lr[m] [bn 'bdmlk l'l]y lršp mkl k šm' ql ybrk

[---]-ilpa-si-le-wo-selmi-li-ki-ya-to-no-selke-ti-onelka-e-ta-li-onelpa-

si-le-u

[---]-me-na-nelto-pe-pa-me-ro-nelne-wo-so-ta-taselto-na-ti-ri-ya-ta-

nelto-te-ka-te-se-ta-sel o-wa-na-xe

[---]-o-a-pi-ti-mi-li-ko-nelto-a-po-lo-nilto-a-mu-

ko-lo-ila-po-i-wo-ilta-

sele-u-ko-la-se

[e]-pe-tu-keli-tu-ka-ila-ga-ta-il

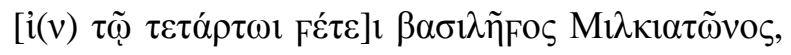

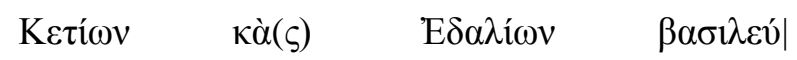

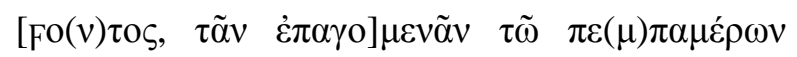

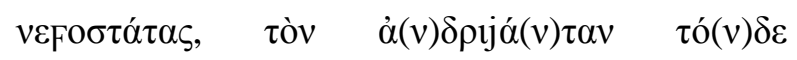

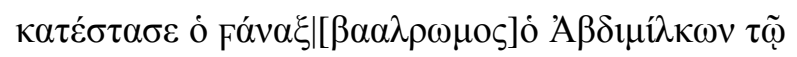

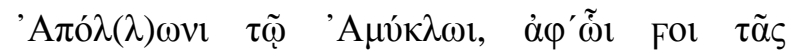
$\varepsilon \hat{\chi} \chi \omega \lambda \tilde{\alpha} \varsigma$

$[\dot{\varepsilon}] \pi \dot{\varepsilon} \tau u \chi \varepsilon \cdot i(v) \tau u ́ \chi \alpha \iota \dot{\alpha} \gamma \alpha \theta \tilde{\alpha} \mathrm{l}$.
«[Le jour ? du mois de ?] en l'année 4 du roi mlkytn, r[oi de Kition et Idalion cette statue est ce qu'a donné et érigé notre seigneur $b \operatorname{lr}[\mathrm{m}$, fils de 'bdmlk à] son dieu, à $r \check{s} p m k l$, parce qu'il a écouté sa voix. Puisse-t-il le bénir !»

«[Dans la quatrième année] du roi Milkyatôn régnant sur Kition et Idalion, au dernier (jour) de la période de cinq jours des jours intercalaires, le prince [Baalrômos], le fils de Abdimilkôn, a dédié cette statuette à Apollon Amyklos, de qui il a obtenu pour lui-même l'accomplissement de son vœu. À la Bonne Fortune »

L'inscription gravée sur la base d'Idalion livre la seule attestation en grec du nom du roi mlkytn. La séquence syllabique mi-li-ki-ya-to-no-se exprime sans aucun doute un génitif qu'O. Masson transcrit comme Mi $\lambda \kappa i j \alpha ́ \theta \omega v o \varsigma$, d'après l'étude de J. Friedrich ${ }^{42}$ sur l'onomastique de la présente inscription, mais qu'il vaut mieux rendre par Mı $\lambda \kappa \iota \alpha \tau \omega v o \varsigma$, comme cela a été corrigé plus tard, dans la troisième édition de la Phönizisch-punische Grammatik ${ }^{43}$. En fait la dernière proposition s'accorde avec les règles de transcription des consonnes entre les deux langues, à cette époque, telles qu'elles ont été observées et décrites par M. G. Amadasi Guzzo en 199944. Dans le cas présent, l'on peut noter qu'entre le $\mathrm{V}^{\mathrm{e}}$ et le III' $\mathrm{s}$. av. J.-C., le grec transcrit systématiquement au moyen de $\tau$ les deux dentales phéniciennes $t$ et $t$. Le nom du roi a été absorbé par la déclinaison en nasale, suivant le modèle

\footnotetext{
${ }^{42}$ Friedrich $1953:$ 90-92.

${ }^{43} P P G^{3}$ 37b.

${ }^{44} P P G^{3} 37$.
} 
A $\gamma \alpha \dot{\alpha} \theta \omega v,-\omega v o \varsigma$. Ce choix respecte la phonétique du nom de départ, qui se compose du nom divin $m l k^{45}$ suivi de la $3^{\mathrm{e}}$ pers. m. s. du parfait Qal de la racine $\mathrm{YTN}^{46}$ «donner ». Ce dernier élément verbal est censé avoir une vocalisation de la deuxième syllabe en $\overline{0}^{47}$, ce qui représente un segment phonétique prêt pour être inséré dans la classe morphologique grecque évoquée. La forme syllabique mi-li-ki-yato-no-se pourrait faire penser à la prononciation d'une voyelle de liaison entre les deux éléments composant l'anthroponyme, mlk et ytn, c'est-à-dire *milkiyatôn. Mais à cause d'une spécificité graphique de l'écriture chypriote, qui note souvent mais pas systématiquement les /y/ en fonction de glide entre une voyelle /i/ suivie d'une autre voyelle ${ }^{48}$, toute reconstruction demeure ambiguë.

\section{Les anthroponymes bâtis sur le théonyme $d^{6} m$ : $C I S$ I, 115}

En nous déplaçant dans un secteur exclusivement grec, celui de la Grèce continentale, notre attention est attirée par l'épitaphe bien connue d'Antipatros d'Ascalon ${ }^{49}$.

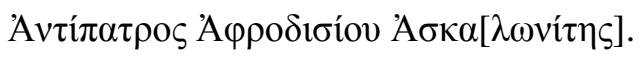

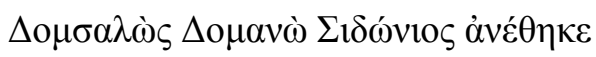

'nk šmy bn 'bd‘štrt ’šqlny

’š yṭn’t 'nk d'mṣlḥ bn d'mḥn’ șdny
«Antipatros fils d'Aphrodisios d'Ascalon. Domsalôs fils de Domanôs de Sidonien a consacré (cette stèle) »

« Je suis šmy, fils de 'bd ‘̌trt, l’Ascalonite. (Voici ce) que moi j'ai érigé d'mșlh, fils de d'mhn', le Sidonien»

Le bas-relief, dont la composition sort des schémas de la tradition figurative grecque, et l'épigramme qui, loin de clarifier la signification des images, les rend plus ambiguës, donnent à ce monument son aspect thaumastós. Néanmoins, la stèle d'Antipatros est pourvue d'une épitaphe classique, qui livre quatre anthroponymes phéniciens adaptés différemment à la langue grecque. En fait, le nom du défunt et son patronyme semblent être passés à travers le filtre de l'interprétation,

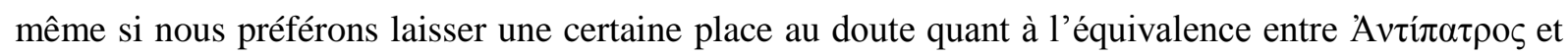

\footnotetext{
${ }^{45}$ Benz $1972: 344$.

${ }^{46}$ DNWSI : 478-480.

${ }^{47} \mathrm{Il}$ s'agit du passage en [o] d'une voyelle [a] accentuée qui s'est allongée secondairement, $c f . P P G^{3} 78 \mathrm{a}, 78 \mathrm{c}$.

${ }^{48}$ Voir Masson $1961: \S 28,2$ et $\S 36$; Egetmeyer $2010: 119$ et 126-128.

${ }^{49}$ CIS I, $115=I G$ II $2836=I G$ II $^{2} 8388=K A I 54$. Nous ne prenons en considération ici que l'épitaphe et la dédicace du monument également pourvu d'une épigramme qui, toutefois, ne concerne pas notre enquête. Une étude approfondie de l'épigramme et du relief a été récemment faite par O. Tribulato (Tribulato 2013: 459-486). Voir aussi C. Bonnet, Les Enfants de Cadmos. Le paysage religieux de la Phénicie hellénistique, Paris, De Boccard 2014, p. 462-471.
} 
$\check{s} m y$, qui n'est pas évidente ${ }^{50}$. Son père, qui s'appelait ' $b d$ 'štrt a en revanche trouvé un équivalent

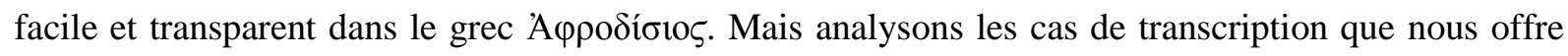
l'épitaphe. Le nom du Sidonien qui s'est chargé de l'érection du monument funéraire et celui de son père sont rendus en grec phonétiquement, sans interprétation. Ces derniers noms se composent d'un théonyme très peu attesté dans l'onomastique phénicienne et punique, $d^{\prime} m^{51}$. Celui-ci semble être une divinité secondaire du panthéon phénicien, attestée uniquement par quelques inscriptions ${ }^{52}$. Cet état de choses a empêché toute tentative d'interprétation d'un point de vue religieux. Les deux noms sont des hapax en phénicien comme en grec, que l'on peut traduire, selon l'ordre d'apparition dans le texte, par « $d^{\prime} m$ a rendu prospère » et «d'm lui a rendu grâce ». En adaptant le phénicien, le grec garde la phonétique de ce dernier mais, en même temps, recourt à ses catégories grammaticales propres : c'est ainsi que d'mṣlh est rendu par un nominatif $\Delta \mathrm{o} \mu \sigma \alpha \lambda \omega \grave{\omega}$, une forme qui, d'une part, garde le vocalisme du mot de base souligné par la notation $\omega$ du grec en correspondance de ō de la deuxième syllabe du parfait Qal $3^{\text {e }}$ pers. m. s. du phénicien et, de l'autre, marque le nominatif par un sigma final. Le deuxième nom, $d^{\prime} m h h^{\prime}$ ', pose des problèmes dès le phénicien. Le théonyme $d^{\prime} m$ est en effet uni à la $3^{\mathrm{e}}$ pers. m. s. du parfait Qal de la racine $\mathrm{HCNN}^{53}$, suivie du suffixe personnel accusatif de la $3^{\text {e }}$ pers. m. s. : $d^{\prime} m+h h+$ '. Le suffixe personnel est ainsi noté avec un 'aleph. Ce qui est remarquable, c'est qu'en phénicien on ne connaissait pas auparavant ce type de notation pour les suffixes personnels en voyelles. Il s'agit d'un système orthographique attesté dans la phase punique de la langue, qui la caractérise $^{54}$. En conséquence, nous accueillons ici l'hypothèse de M. G. Amadasi Guzzo ${ }^{55}$, qui songe à un cas de notation vocalique jusqu'à présent occasionnel en phénicien. La transcription grecque indique fidèlement la prononciation phénicienne et confirme aussi la reconstruction du suffixe accusatif de la $3^{\mathrm{e}}$ pers. m. s. D'ailleurs, la syntaxe grecque demande ici le patronyme au génitif, ce qui

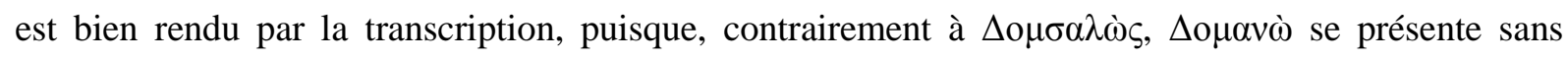
sigma final et se laisse interpréter comme un génitif. Le rédacteur aurait ainsi adopté un expédient assez fonctionnel, en distinguant les deux anthroponymes, qui se succèdent dans le texte, au moyen d'un sigma marquant le nominatif, et d'aucune marque en transcrivant le nom qui n'a pas fonction

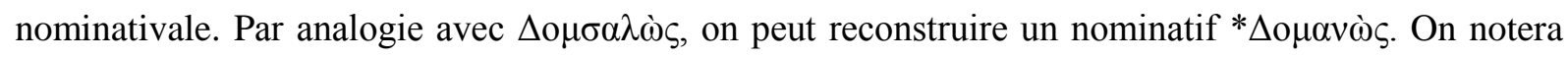
une fois encore qu'à la pharyngale $h$ le grec ne fait correspondre aucun phonème.

\section{Le vrai nom d'Aбє $\pi \tau$ dans $C I S$ I, 119}

\footnotetext{
${ }^{50}$ Pour une réflexion sur la correspondance possible entre le nom du défunt en grec et en phénicien, $c f$. Bonnet $1990: 42-43$.

${ }^{51}$ Benz $1972: 108$ et 301 .

${ }^{52}$ Sur cette divinité, voir Lipiński $1995: 190-192$

${ }^{53}$ Voir Benz $1972: 313$ et Amadasi $1991: 4-5$.

${ }^{54}$ Huehnergard $1991: 183-192 ; P P G^{3} 112$.

${ }^{55}$ Amadasi \& Bonnet $1991: 5$.
} 
Un nombre assez considérable de stèles funéraires trouvées en Attique et remontant au $\mathrm{IV}^{\mathrm{e}}$-III ${ }^{\mathrm{e}}$ siècle av. J.-C. atteste la présence de Sidoniens. L'épitaphe bilingue d'une femme ensevelie au Pirée nous fait connaître son origine sidonienne, cet élément ethnique étant enregistré dans les deux versions de l'inscription gravée sur sa tombe.

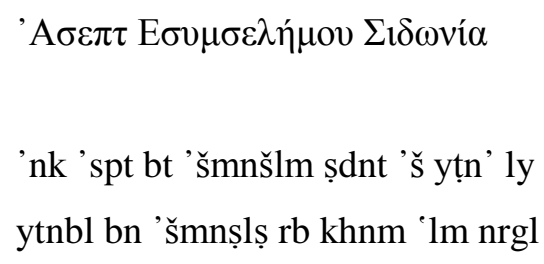

« Asept fille d'Esymselèmos, sidonienne »

«Je suis 'spt, fille d'šmnšlm, de Sidon. (Ceci est ce) qu'a érigé pour moi ytnbl, fils de 'šmnșlṣ, grand-prêtre du dieu Nergal »

La version grecque de l'inscription n'enregistre que le nom de la défunte et son patronyme, en laissant le nom du commanditaire de la stèle et son propre patronyme sans correspondant en grec. Le nom de la défunte est tout simplement transcrit en grec; il n'est ni interprété ni adapté morphologiquement. Ce type d'adaptation est très rare : la documentation disponible nous offre seulement deux autres cas dans une inscription trilingue provenant de Leptis Magna ${ }^{56}$. L'inscription ayant \& té gravée en scriptio continua, les éditeurs du texte ont proposé deux lectures différentes du

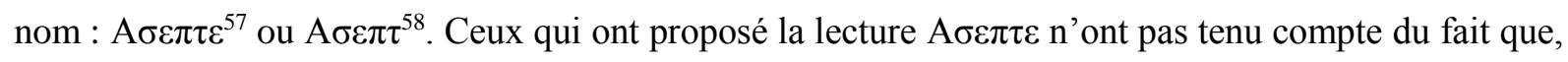
quand les noms phéniciens sont adaptés en grec au moyen de transcriptions, ce processus obéit aux règles grammaticales grecques, à travers des adaptations morphologiques. Comme on l'a vu pour les cas analysés jusqu'ici, les nouvelles formations adoptent un aspect morphologique usuel en grec. A $\sigma \varepsilon \pi \tau \varepsilon$, en revanche, avec la notation d'un epsilon final, se situe en dehors de toute catégorie grammaticale grecque. C'est pourquoi, nous croyons pouvoir affirmer que le nom de la défunte dans le

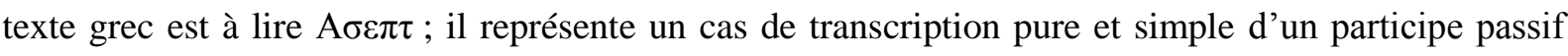
phénicien de la racine ' $\mathrm{SP}^{59}$ « rassembler», qui serait *asipt. La signification des noms formés sur cette racine est à notre avis bien saisie par Noth ${ }^{60}$, qui met en lumière le double sens de la racine « rassembler», à savoir «rassembler pour éliminer» et «rassembler pour recueillir». Nous le suivons, en attribuant à la racine 'SP le sens de «prendre avec soi, prendre sous sa protection». Ainsi, Asept serait celle qui a été « recueillie » et accueillie parmi les adeptes d'une certaine divinité ou par la divinité elle-même.

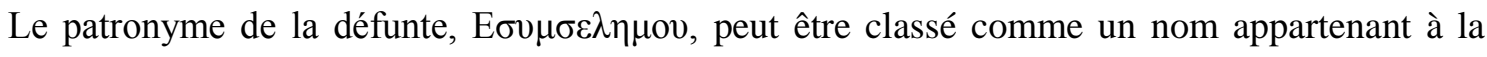
déclinaison thématique, grâce à la marque d'un génitif en -ov. La transcription de cet anthroponyme

\footnotetext{
${ }^{56}$ IPT 12.

57 CIS I, 119; IG II 3319 (U. Koehler); IG II $^{2}$ 10271(J. Kirchner): mais alors le patronyme serait $\Sigma v \mu \sigma \varepsilon \lambda \eta \dot{\mu o v} ?$

${ }^{58}$ KAI 59 (W. Röllig).

${ }^{59}$ DNWSI : 89.

${ }^{60}$ Noth $1928: 181-182$.
} 
en grec nous révèle sa prononciation réelle, (Esum- à la place de Eshmun- qu'on attendrait ; selēm rendant *šillēm, comme [Asept rend *asipt]), bien sûr enfermée et limitée par le système phonématique d'une langue différente, non sémitique. En phénicien, le nom est enregistré normalement suivant sa graphie historique. En lisant le grec, il est clair que 'šmnšlm a subi une réduction du corps phonétique, vraisemblablement suite à un mécanisme de simplification qui a porté sur les syllabes pré-toniques.

\section{Le témoignage épigraphique d'Abdalonymos de Sidon à Cos}

En 1982, parmi les débris d'un terrain transportés par un camion, le hasard a livré un bloc de marbre inscrit en grec et en phénicien, qui, dans la dédicace, présente la seule occurrence épigraphique du nom du roi Abdalonymos de Sidon. Jusqu'à cette découverte son existence n'était connue que par les récits de quelques auteurs classiques ${ }^{61}$, qui en parlent de façon contradictoire. Ayant été remployée, probablement comme élément architectural, la pierre est dans un état déplorable qui empêche notamment le déchiffrement complet de la partie phénicienne occupant la partie inférieure du support. Toutefois, les multiples efforts de M. Sznycer ${ }^{62}$ ont permis de lire une partie significative du texte phénicien, tandis que M. G. Amadasi Guzzo ${ }^{63}$ a pu récemment proposer une interprétation d'une partie du texte qui semble convaincante :

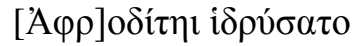

$[\Delta \mathrm{ió}] \tau 1 \mu \mathrm{o}$ 'A $\beta \delta \alpha \lambda \omega v v_{\mu o v}$

$[\Sigma 1 \delta] \tilde{\omega} v_{0 \varsigma} \beta \alpha \sigma 1 \lambda \dot{\varepsilon} \omega \varsigma$

$[\dot{v} \pi] \grave{\varepsilon} \rho \tau \tilde{\omega} v \pi \lambda \varepsilon \cos \tau \omega \nu$

lrbty l'štrt p`lt t(?)1 z’ [š ytn/ndr ---]

bn mlk 'bd'lnm mlk șdnym 'l hy $\mathrm{kl}$ des Sidoniens, pour la vie de [tous les [mlḥm/ḥblm]

'̌s(?).....'1 kl mš't '̌s 1 'šmn $\ln \ldots$
«Diotimos, fils d'Abdalônymos roi de Sidon, a dédié (cette stèle) à Aphrodite, pour les navigateurs »

«À sa dame à ‘̌strt, ceci est l'ouvrage de ...qu'a donné/dédié [---] fils du roi 'bd’lnm, roi marins/navigateurs]/ qui( ?)...pour ( ?) toutes les $m \check{s} ' t$ que »

Le nom du roi Abdalônim signifie en phénicien «serviteur des dieux », en se composant du mot $b d$ «serviteur », suivi de la forme plurielle de ' $l$ « dieu ». Il est présent dans les deux versions de l'inscription. En grec, il a été reproduit phonétiquement, avec pour suffixe la terminaison du nominatif des noms thématiques. Il s'agit d'un anthroponyme qui ne compte pas de nombreuses occurrences

\footnotetext{
${ }^{61}$ Diod. XVII, $46-47$; Just. XI, 10 ; Plut., De Alex. Fort., II, 8.

${ }^{62}$ Kantzia \& Sznycer $1986: 17-30$; Sznycer $1999: 103-115$.

${ }^{63}$ Amadasi 2013 : 153-158.
} 
dans le répertoire onomastique phénicien et punique ${ }^{64}$, mais il est attesté encore en milieu grec, dans une inscription bilingue ${ }^{65}$ provenant de Démétrias de Thessalie. En l'occurrence, le nom grec qui lui

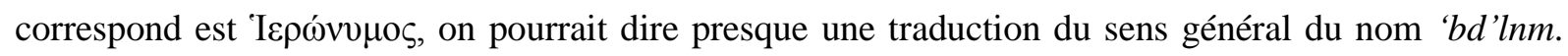
Au contraire, dans le cas du roi de Sidon, on a gardé une graphie phonétique du nom, en l'adaptant à la morphologie grecque ; peut-être le nom devait rester le même dans les deux langues, s'agissant d'un personnage de rang royal. L'auteur de la dédicace est son fils, dont le nom phénicien ne nous est pas parvenu, à cause de la brisure de la pierre. Dans la partie grecque, en revanche, il est mentionné et

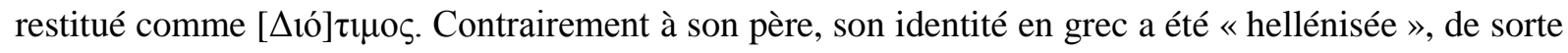
que l'on ne peut qu'essayer de deviner son nom en phénicien. L'on ne s'éloignera pas trop de la vérité, en songeant à un anthroponyme construit à partir du théonyme $\mathrm{Ba}{ }^{\circ} \mathrm{al}$. En effet, Ba'al, qui le plus souvent se trouve au sommet de la hiérarchie religieuse des panthéons phéniciens, est associé par l'iconographie soit à Poséidon soit à Zeus, alors que l'anthroponymie révèle une correspondance constante avec Zeus ${ }^{66}$.

\section{Conclusions}

L'ensemble de la documentation analysée offre des données qui semblent révéler une certaine régularité, presque une règle, présidant à la transcription en grec des anthroponymes phéniciens. Dans

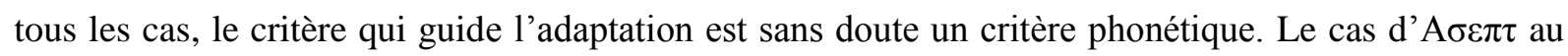
Pirée constitue l'exemple le plus éloquent de ce critère, même s'il est un peu à part, du fait qu'il est simplement transcrit et non adapté à un schéma morphologique grec. En revanche, dans tous les autres cas, on remarque qu'à partir d'une base constituée par la prononciation du corps phonétique du nom de départ, l'adaptation s'accomplit en ajoutant des terminaisons spécifiques. Le choix des terminaisons morphologiques, qui permettent aux anthroponymes phéniciens d'acquérir un aspect grec, de s'helléniser en quelque sorte, se fait en respectant deux paramètres. D'une part, la réalisation phonétique du nom phénicien peut en déterminer l'insertion dans une classe morphologique précise, en offrant un segment de sons reconnaissable par les locuteurs grecs. On songe, par exemple, au cas de mlkytn/ Mi $\lambda \kappa \iota \alpha \tau \omega v o \zeta$, dans la mesure où la prononciation de l'anthroponyme phénicien permet de l'analyser et de l'adapter comme un nom grec en -ōn. D'autre part, c'est la langue grecque qui sélectionne un système d'adaptation, sans négliger l'aspect phonétique du nom de départ. On peut

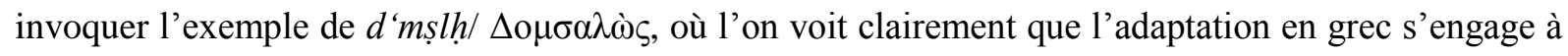
reproduire la chaîne phonétique de l'anthroponyme phénicien, sans pour autant renoncer à l'insérer dans une classe grammaticale grecque, au moyen d'un sigma final marquant un nominatif.

\footnotetext{
${ }^{64}$ Benz 1972 : 149.

${ }^{65}$ Masson $1969: 698$

${ }^{66}$ Baslez 1986 : 296-299
} 
Dans certain cas, c'est une variété dialectale de la langue grecque, avec sa morphologie spécifique, qui a accueilli les noms phéniciens. On songe ici au cas de leur insertion dans la déclinaison chypriote des noms en en $-\tilde{\alpha} \varsigma,-\tilde{\alpha}(F) \circ \varsigma(>\tilde{\alpha} o \varsigma)$. Ce sont en général les hypocoristiques phéniciens qui sont insérés dans cette déclinaison. Or, sans entrer dans la question de la prononciation des suffixes hypocoristiques phéniciens, et sans exclure 1 'hypothèse ${ }^{67}$ qu'une relation phonétique ait suscité une correspondance entre les suffixes hypocoristiques du phénicien et les terminaisons de la déclinaison chypriote des noms en- $\tilde{\alpha},-\tilde{\alpha}(F) \circ \varsigma(>\tilde{\alpha} \circ \varsigma)$, on peut néanmoins observer que ${ }^{*} \Sigma \varepsilon \sigma \mu \tilde{\alpha} \varsigma$,

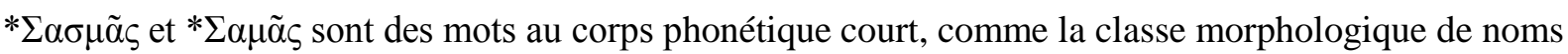
hypocoristiques. En fait, à Chypre, les noms qui appartiennent à cette catégorie sont surtout des sobriquets ${ }^{68}$. L'étendue du corps phonétique de ces quelques anthroponymes phéniciens semble un élément significatif pour leur insertion dans la catégorie grammaticale évoquée. En effet, l'autre occurrence du théonyme ssm, à savoir abdssm/ A $\psi \alpha \sigma \mu o \varsigma$, se situe en dehors de ce cadre morphologique, car il ne s'agit pas d'un nom raccourci, mais à l'inverse d'un nom complet. Tout comme 'A $\beta \delta \alpha \lambda \omega ́ v v \mu o \varsigma$ à Cos, $\mathrm{A} \psi \alpha \sigma \mu \mathrm{o}$ à Tamassos a reçu une adaptation qu'on pourrait qualifier de classique, du fait que, en principe, la classe des noms thématiques se montre très réceptive pour accueillir les nouvelles formations onomastiques des Phéniciens de Grèce.

Cette étude a permis de dresser un tableau raisonné des transcriptions des noms phéniciens en grec dans le cas des inscriptions bilingues gréco-phéniciennes. Dans le périmètre restreint de l'enquête menée, bien d'autres questions émergent : les anthroponymes phéniciens «hellénisés » sont-ils des vrais noms personnels? En d'autres termes : les noms phéniciens transcrits en grec étaient-ils entrés dans l'usage commun, étaient-ils utilisés dans les milieux grecs où les Phéniciens s'étaient établis ? La régularité des mécanismes d'adaptations, la récurrence de phénomènes qui se répètent de façon assez mécanique, comme les terminaisons qui s'ajoutent aux noms phéniciens tel qu'ils étaient probablement prononcés, nous font pencher pour une première réponse négative. Mais la question est délicate et complexe et elle demandera à être mise à l'épreuve des données issues d'un plus grand nombre et d'une plus grande variété de documents.

Maria BIANCO

Laboratoire CRISES-EA 4424

Labex ARCHIMEDE, programme IA-ANR-11-LABX-0032-01

Université Paul-Valéry, Montpellier

Route de Mende, 34199 Montpellier Cedex 5

maria.bianco@univ-montp3.fr

\footnotetext{
${ }^{67}$ Nous envisageons une étude d'ensemble sur les suffixes hypocoristiques en phénicien et leur possible réalisation phonétique en grec.

${ }^{68}$ Egetmeyer $2010: 354$
} 


\section{Abréviations}

BDB Brown (F.), Driver (F.S.), Briggs (C.), The Brown-Driver-Briggs Hebrew and English Lexicon, Peabody MA 2000.

CIS Corpus Inscriptionum Semiticarum, Pars Prima. Inscriptiones Phoenicias continentes, Paris 1881 et ss.

DCPhP Lipiński (E.), Dictionnaire de la civilisation phénicienne et punique, Turnhout 1992.

DNWSI Hoftijzer (J.) \& Jongeling (K.), Dictionary of the North-West Semitic Inscriptions (Handbuch der Orientalistik, 21), Leiden 1995.

ICS Masson (O.), Les Inscriptions chypriotes syllabiques, Paris 1961.

IPT Levi Della Vida (G.) \& Amadasi Guzzo (M. G.), Iscrizioni puniche della Tripolitania (19271967), Roma 1987.

KAI Donner (H.) \& Röllig (W.), Kanaanäische und aramäische Inschriften. Wiesbaden 1966- 2002. 3 Vols. I ${ }^{5}$ 2002, II ${ }^{2}$ 1966, III 1969.

KTU Dietrich (M.), Loretz (O), Sanmartín (J.), Die keilalphabetischen Texte aus Ugarit. Kevelaer/Neukirchen/Vluyn 1976.

OGIS Dittenberger (W.), Orientis Graeci Inscriptiones Selectae. 2 vols. Leipzig 1903-1905.

PPG $^{3} \quad$ Friedrich (J.), Röllig (W.), Phönizisch-punische Grammatik (Analecta Orientalia 55; 3. Auflage, neu bearbeitet von M.G. Amadasi Guzzo unter Mitarbeit von W.R. Mayer), Roma 1999

RÉS Répertoire d'épigraphie sémitique, Paris 1900 et ss.

\section{Bibliographie}

Amadasi Guzzo (M. G.) \& Bonnet (C.)

1991 «Anthroponymes phéniciens et anthroponymes grecs : remarques sur leur correspondances », SEL, 8, 1-21.

Amadasi Guzzo (M. G.)

2013 « Notes sur quelques inscriptions phéniciennes provenant de l'Egée » dans Fr. BriquelChatonnet, C. Fauveaud et I. Gajda (éd.), Entre Carthage et l'Arabie heureuse. Mélanges offerts à François Bron, (Orient \& Méditerranée), Paris, 163-176.

Benz (F. L.)

1972 Personal Names in the Phoenician and Punic Inscriptions (Studia Pohl, 8), Rome.

Berger (Ph.)

1887 «La seconde inscription bilingue de Tamassus », CRAI, 2, 187-201.

Bonnet (C.)

1990 «La terminologie phénico-punique relative au métier de lapicide et à la gravure des textes », SEL, 7, 111-122.

Bonnet (C.)

2004 «Le roi et la déesse. À propos de la dédicace grecque à Ptolémée et Aphrodite de la grotte de Wasța, près de Tyr », SEL, 21, 125-140.

Briquel-Chatonnet (F.)

1995 «Quelques remarques sur l'onomastique des Phéniciens d'après les inscriptions grecques », ACFP III, 203-209. 
Caquot (A.) \& Masson (O.)

1968 «Deux inscriptions phéniciennes de Chypre », Syria, 45, 295-321.

Clermont-Ganneau (Ch.)

1888 Recueil d'archéologie orientale, I, Paris.

Egetmeyer (M.)

2010 Le dialecte grecque ancien de Chypre, 2 vols., Berlin/New York.

Espagne (M.) \& Werner (M.)

1987 «La Construction d'une référence culturelle allemande en France : Genèse et histoire (1750 1914) », Annales, économies, sociétés, civilisations, 4, 969-992.

Friedrich (J.)

1953 «Phonizische Namen in kyprischer Silbenschrift », WZKM, 53, 90-92.

Gjerstad (E.)

1946 «Four Kings », Opuscula Archaeologia, 4, 21-24.

Huehnergard (J.)

1991 « The Development of the Third Person Suffixes in Phoenician », Maarav, 7, 183-94.

Kantzia (Ch.) \& Sznycer (M.)

1986 «... TIMO $\Sigma$ АВГА $\Lambda \Omega N Y M O Y \Sigma I \Gamma \Omega N O \Sigma$ BA $\Sigma \mathrm{I} \Lambda \Delta \Omega \Sigma$. La partie

phénicienne de l'inscription bilingue gréco-phénicienne de Cos », $\mathrm{AD}, 35,1-30$.

Lagrange (M.-J.)

1902 «Deux hypogées macédo-sidoniens à Beit-Djebrîn (Palestine) », CRAI, 5, 497-505.

La Regina (A.)

1989-1990 «Il vaso con iscrizione dalla tomba 482 dell'Osteria dell'Osa », dans A.M. Bietti Sestieri, A. De Santis, A. La Regina (éd.), «Elementi di tipo cultuale e doni personali nella necropoli laziale di Osteria dell'Osa », ScAnt, 3-4, 83-88.

Lipiński (E.)

1995 Dieux et déesses de l’Univers Phénicien et Punique, (Studia Phoenicia, 14 ; Orientalia Lovaniensia Analecta, 64), Leuven.

Masson (O.)

1958 « Notes d'onomastique chypriote: le nom ki-li-ka-se et les noms en -as à

Chypre », dans Sybaris. Festschrift H. Krahe, 67-72.

Masson (O.)

1961 Les Inscriptions chypriotes syllabiques, Paris 1961

Masson (O.)

1968 «Kypriaka », BCH, 92, 375-409.

Masson (O.) \& Sznycer (M.)

1972 Recherches sur les Phéniciens à Chypre (Hautes études orientales, 3), Genève-Paris.

Morpurgo Davies (A.) 
1988 «Problems in Cyprian Phonology and Writing » dans J. Karageorghis, O. Masson (éd.), I The History of the Greek Language in Cyprus, Nicosia, 99-130.

Sznycer (M.)

1999 « Retour à Cos », Semitica, 49, 103-116.

Tribulato (O.)

2013 «Phoenician Lions : The Funerary stele of the Phoenician Shem/Antipatros », Hesperia, 82, 459-486. 